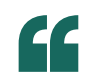

With 13 individual components, this is the most complex system we have assembled so far

\title{
A reaction regulation network
}

Molecular switches have frequently been used to turn on and off catalytic reactions, but only a small number have been shown to be capable of regulating different types of reaction.

Writing in the Journal of the American Chemical Society, Michael Schmittel and co-workers from the University of Siegen describe the construction of a 13-component network in which two different reactions - an organocatalysed Michael addition and a coppercatalysed cycloaddition - are turned on and off in response to an added stimulus. "We were motivated by the way living systems make decisions," says Schmittel. "For example, cells maintain homeostatic conditions through a complex network of reactions that are up- and down-regulated and feedback."

Schmittel and his team have previously reported the assembly of multicomponent nanorotors that can release an organocatalyst into solution. They showed that the concentration of free organocatalyst, and thus the rate of the reaction it mediates, was correlated to the rotation speed of their rotor. In their latest work, they designed a responsive system in which two distinct rotor systems would selfassemble from their components. The rotors are heteroleptic complexes of either copper(I) or zinc(II) coordinated by a stator (S) that bears two pendant zinc porphyrins and one of two different rotor arms (R1 and R2) that coordinate the central metal and present a picoline or pyridine, respectively, that can bind to either of the porphyrins in the stator. A reservoir of the two rotors is provided in the form of bis-copper species coordinated by two molecules of R1 or R2.

The addition or removal of zinc ions leads to the formation of two distinct network states (NetStates). In NetState I (in the absence of zinc), coordination of $\mathrm{R} 2$ in the rotor is thermodynamically favoured. The slow rotation of this assembly releases a small amount of an aza-crown ether into solution, which catalyses the addition of thiophenol to a Michael acceptor.

The addition of zinc(II) prompts an interconversion of the rotor assemblies, leading to NetState II this time favouring the zinc rotor in combination with the stator and the picoline containing rotor arm (R1). The rotational speed of this

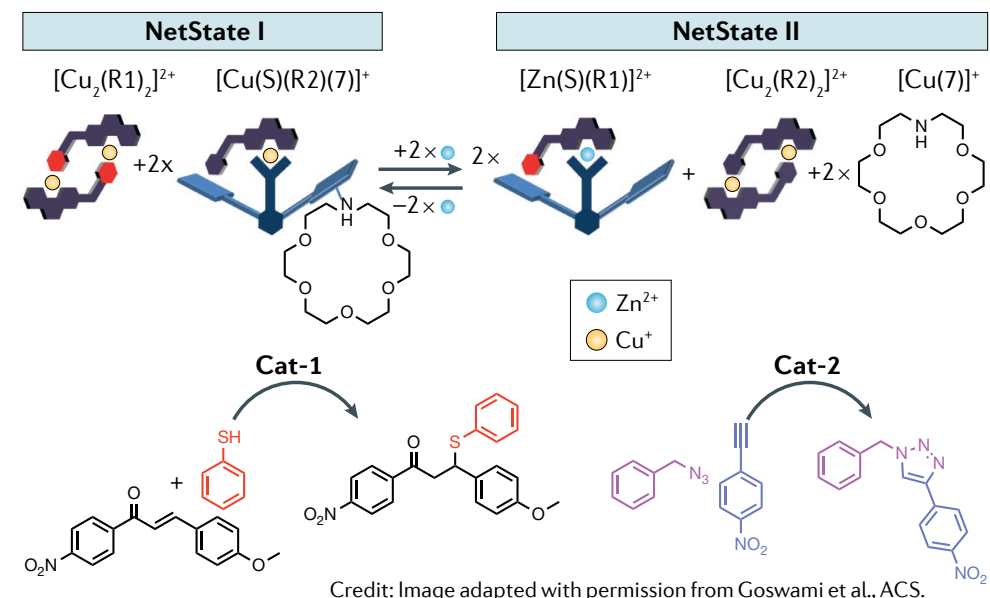

system is higher, releasing a greater concentration of the aza-crown ether into solution where it coordinates to copper(I). In this form, the aza-crown ether does not catalyse the Michael reaction but it is, instead, a competent catalyst for a Huisgen cycloaddition.

Sequestration of the zinc(II) by the addition of an equivalent of hexacyclen returns the system to NetState I - the cycloaddition reaction is now turned off and the organocatalytic Michael reaction reactivated.

"With 13 individual components, this is the most complex system we have assembled so far," says Schmittel. "We think it unlikely that many people would predict the decision-making processes triggered by adding/ removing zinc and we think this is in some ways similar to the intricate networks found in nature." Indeed, in isolation, the zinc-based system has a higher rate of rotation, which would act to increase the concentration of organocatalyst in solution and thus speed up the Michael addition. In the ensemble system, however, the organocatalyst is sequestered by released copper so the Michael addition is switched off.

"We think that our work shows that manmade systems chemistry — like systems biology — will be suitable for generating powerful information systems," says Schmittel. "Moreover, we think it shows that it is time to move beyond stand-alone molecular devices to develop novel functions that are only feasible by building smart intricate networks."

Stephen G. Davey

ORIGINAL ARTICLE Goswami, A. et al. Switching dual catalysis without molecular switch: using a multicomponent information system for reversible reconfiguration of catalytic machinery. J. Am. Chem. Soc. 141, 15656-15663 (2019) RELATED ARTICLE van Dijk, L. et al. Molecular machines for catalysis. Nat. Rev. Chem. 2, 0117 (2018) 